\title{
Synthesis of disubstituted- and deoxydisubstituted- derivatives of $\alpha$-D-xylofuranose as anticancer agents
}

\author{
Nawal Kishore, ${ }^{a}$ Neelima Sinha, ${ }^{a}$ Sanjay Jain, ${ }^{*}{ }^{a}$ Ram Shankar Upadhayaya, ${ }^{a}$ \\ Ramesh Chandra ${ }^{b}$, and Sudershan K. Arora $*^{a}$ \\ ${ }^{a}$ Medicinal Chemistry Division, New Chemical Entity Research, Lupin Research Park \\ 46/47A, Village Nande, Taluka Mulshi, Pune 411 042, Maharashtra, India \\ ${ }^{b}$ Bundelkhand University, Jhansi 284 128, Uttar Pradesh, India \\ E-mail: sanjayjain@lupinpharma.com (SJ), sudershanarora@,hotmail.com (SKA)
}

(received 25 Nov 04; accepted 29 Dec 04; published on the web 03 Jan 05)

\begin{abstract}
Several disubstituted- and deoxydisubstituted- derivatives of $\alpha$-D-xylofuranose have been synthesized and evaluated on various in vitro human cancer cell lines. In primary in vitro screening, seven derivatives expressed anticancer activity.
\end{abstract}

Keywords: Synthesis, aminoalkyl derivatives, xylofuranose, anticancer activity

\section{Introduction}

A large number of compounds with diverse chemical structure and molecular weight are reported to be effective anticancer agents. ${ }^{1-15}$ Among them, several branched-chain sugar derivatives, ${ }^{11-15}$ as well as some low molecular weight sugar derivatives with aminoalkyl appendages, ${ }^{1-4}$ have shown potent anticancer activity. In continuation of our efforts to find potent anticancer agents and on the basis of the correlation of anticancer activity in vitro, in vivo, and lipophilicity of the compounds of our previous work on aminoalkyl derivatives of sugars, ${ }^{4,16}$ we have synthesized disubstituted- and deoxydisubstituted- derivatives of $\alpha$-D-xylofuranose and evaluated them for anticancer activity.

\section{Results and Discussion}

\section{Chemistry}

Scheme 1 indicates the synthetic route to 3-O-\{3'-(decyloxy)propyl $\}-1,2-O$-isopropylidene-5-O(substituted)- $\alpha$-D-xylofuranose (7a-h) from 1,2:5,6-di- $O$-isopropylidene- $\alpha$-D-glucofuranose (1) ${ }^{17}$. Compound 1 was $O$-alkylated at the $\mathrm{C}-3$ position with 3-chloro-1-propanol in the presence 
of $\mathrm{NaOH}$ at $110{ }^{\circ} \mathrm{C}$ to afford compound 2 in $74 \%$ yield, which was further $O$-alkylated at C-3' position with 1-bromodecane to give 3-O-\{3'-(decyloxy)propyl $\}-1,2: 5,6$-di- $O$-isopropylidene- $\alpha$ D-glucofuranose (3) in good yield.

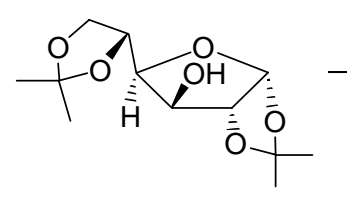

1
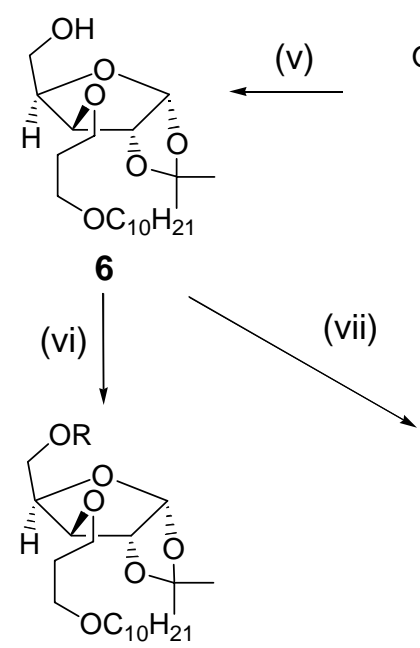

7a-h

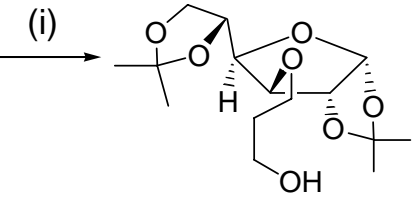

2

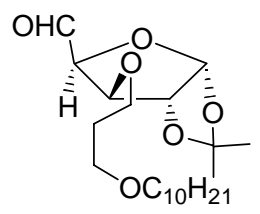

5

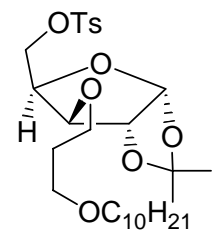

8

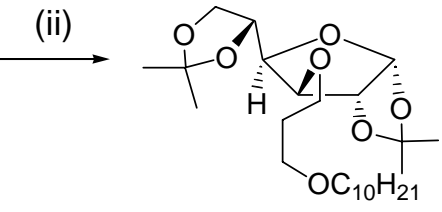

3

(iii)

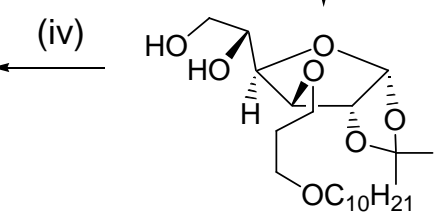

4

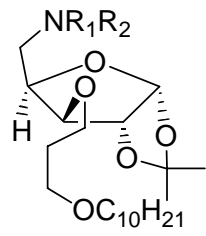

9a-m
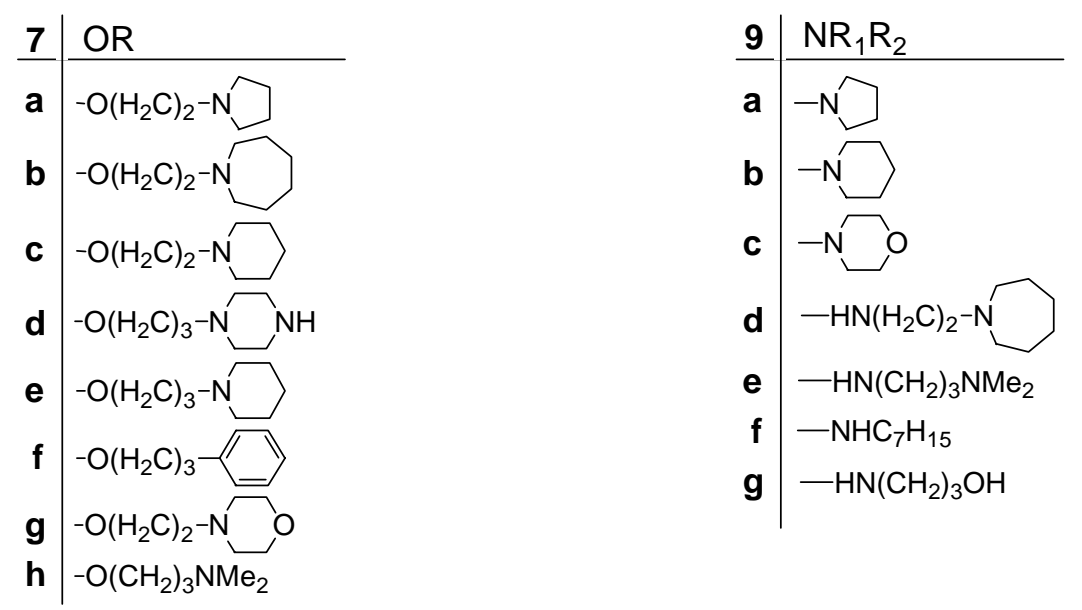

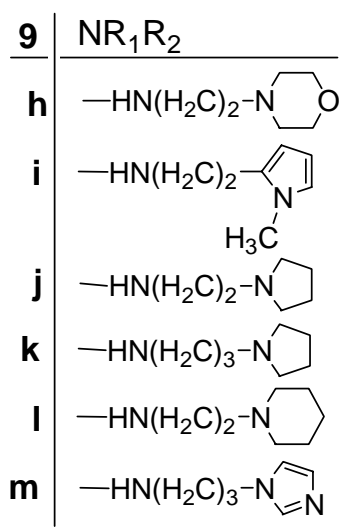

Scheme 1. Synthesis of compounds 7 and 9. (i) $\mathrm{Cl}\left(\mathrm{CH}_{2}\right)_{3} \mathrm{OH}, \mathrm{NaOH}, 110{ }^{\circ} \mathrm{C}, 8$ h. (ii) $\mathrm{C}_{10} \mathrm{H}_{21} \mathrm{Br}$, $\mathrm{NaOH}, 110{ }^{\circ} \mathrm{C}, 10$ h. (iii) $30 \% \mathrm{HClO}_{4}$, THF, $0 \rightarrow 5{ }^{\circ} \mathrm{C}, 6-8 \mathrm{~h}$. (iv) $\mathrm{NaIO}_{4}, \mathrm{H}_{2} \mathrm{O}, 0 \rightarrow 5{ }^{\circ} \mathrm{C}, 6-7 \mathrm{~h}$; (v) $\mathrm{NaBH}_{4}, \mathrm{MeOH}, 0 \rightarrow 5{ }^{\circ} \mathrm{C}, 6-8 \mathrm{~h}$. (vi) $\mathrm{RCl}, \mathrm{NaOH}, 100{ }^{\circ} \mathrm{C}, 7-8 \mathrm{~h}$. (vii) Tosyl chloride, pyridine, $0 \rightarrow 5{ }^{\circ} \mathrm{C}, 6-7 \mathrm{~h}$. (viii) $\mathrm{R}_{1} \mathrm{R}_{2} \mathrm{NH}, 70{ }^{\circ} \mathrm{C}, 6-8 \mathrm{~h}$. 
The selective deprotection of the 5,6-O-isopropylidene group in compound $\mathbf{3}$ led to the formation of diol 4 in excellent yield. In the ${ }^{1} \mathrm{H}$ NMR of compound 4 , both the $\mathrm{OH}$ protons resonated at $\delta 4.70$ as a broad singlet. The oxidative cleavage of vicinal diol 4 gave the aldehyde $\mathbf{5}$ in excellent yield. The signal for the aldehydic proton in compound $\mathbf{5}$ appeared at $\delta 9.61$ as a broad singlet in the ${ }^{1} \mathrm{H}$ NMR spectrum. Compound 5 gave the key intermediate $3-O-\left\{3^{\prime}-\right.$ (decyloxy)propyl\}-1,2-O-isopropylidene- $\alpha$-D-xylofuranose (6) in $89 \%$ yield on $\mathrm{NaBH}_{4}$ reduction. The condensation of key intermediate 6 with various $1-(\omega$-haloalkyl)-amines in the presence of a base gave the required compounds $\mathbf{7 a}-\mathbf{h}$ in good yields. The structures of compounds $\mathbf{7 a}-\mathbf{h}$ were assigned on the basis of spectral and analytical data. Thus, the ${ }^{1} \mathrm{H}$ NMR spectra of these compounds, in general, showed a triplet for the terminal $\mathrm{CH}_{3}$ protons between $\delta$ 0.90-0.96, a multiplet between $\delta 1.60-1.79$ for $-\mathrm{OCH}_{2} \mathrm{CH}_{2} \mathrm{CH}_{2} \mathrm{O}-$ protons, signals for $\mathrm{NCH}_{2}$ and $\mathrm{OCH}_{2}$ protons appeared between $\delta 2.28-2.82$ and 3.24-3.80 respectively, all other methylene protons appeared together between $\delta 1.18-1.68$, and 1-CH proton showed a doublet between $\delta$ 5.88-6.00. The mass spectra of compounds $7 \mathbf{a}-\mathbf{h}$ also gave their correct molecular ion peaks as $\mathrm{M}+1$.

The key intermediate 6 was further used to synthesize different 3-O-\{3'-(decyloxy)propyl $\}-5-$ deoxy-5-(substituted)- $\alpha$-D-xylofuranoses $(\mathbf{9 a}-\mathbf{m})$ via tosylation of the primary alcohol $(5-\mathrm{OH})$ by $p$-toluenesulphonyl chloride in pyridine at $0{ }^{\circ} \mathrm{C}$ followed by displacement of the tosylate group with various primary and secondary amines as shown in Scheme 1. The structures of all these compounds were determined on the basis of complementary spectroscopic $\left({ }^{1} \mathrm{H}\right.$ NMR and MS) and analytical data (cf. experimental section).

\section{Pharmacological Evaluation}

Several compounds were submitted to the National Cancer Institute (Bethesda, MD) for screening. For primary in vitro screening, compounds $\mathbf{7 a - h}$ and $\mathbf{9 a}-\mathbf{m}$ were evaluated for their cytotoxic potency on three human cell lines (NCI-H460 lung cancer, MCF7 breast cancer and SF-268 CNS cancer). A compound is considered to be active when it reduces the growth of any of the cell lines to $32 \%$ or less. Among them, seven compounds (7a, 7b, 7c, 7f, 7g, 9c and 9h) were found active (concentration $10^{-4} \mathrm{M}$ ) in primary screening. Details of this in vitro test method and the information, encoded by the activity pattern over all cell lines, have been previously reported. ${ }^{18}$ Further investigation is in progress.

In summary, a series of disubstituted- and deoxydisubstituted- derivatives of $\alpha$-Dxylofuranose was synthesized and evaluated for anticancer activity. The first results confirm the validity of our approach providing practical access to $\alpha$-D-xylofuranose based derivatives possessing in vitro antiproliferative activity against human tumor cells. Evaluation of compounds $7 \mathbf{a}, 7 \mathbf{b}, 7 \mathbf{c}, 7 \mathbf{f}, 7 \mathbf{g}, 9 \mathbf{c}$ and $9 \mathrm{~h}$ on a full panel of 60 human cancer cell lines is underway and the results will be disclosed in due course. 


\section{Experimental Section}

General Procedures. Melting points were determined in open capillaries on a Büchi B-545 melting point apparatus. Compounds were routinely checked for their purity on silica gel $60 \mathrm{~F}_{254}$ TLC plates and their spots were visualized by exposing them to iodine vapors or by charring the plates with $5 \% \mathrm{H}_{2} \mathrm{SO}_{4}-\mathrm{EtOH}$ reagent or by spraying the plates with Dragendorff's reagent. IR spectra were recorded on a Perkin-Elmer spectrum RX series FT-IR spectrophotometer. ${ }^{1} \mathrm{H}$ NMR spectra were recorded on a Bruker Advance DRX $200 \mathrm{MHz}$ instrument as solutions (in $\mathrm{CDCl}_{3}$ ) using TMS as internal reference, and chemical shifts values are expressed in $\delta$ units. Mass spectra were run on an Applied Biosystems API 3000 instrument using direct inlet system under positive ion electrospray ionization source. Elemental analyses were carried out with a Perkin Elmer 2400 analyzer and the values found were within $\pm 0.4 \%$ of theoretical values.

1,2:5,6-Di-O-isopropylidene- $\boldsymbol{\alpha}$-D-glucofuranose (1). This was prepared according to the literature method ${ }^{17}$ in $41 \%$ yield; $\mathrm{mp} 108-109{ }^{\circ} \mathrm{C}$ (lit. $\left.{ }^{17} \mathrm{mp} 109-110{ }^{\circ} \mathrm{C}\right) .{ }^{1} \mathrm{H}$ NMR $\left(\mathrm{CDCl}_{3}\right): \delta$ $1.38(\mathrm{~s}, 6 \mathrm{H}), 1.42(\mathrm{~s}, 6 \mathrm{H}), 2.59$ (br s, 1H), 3.80-4.62 (m, 6H), $5.94(\mathrm{~d}, J=4.0 \mathrm{~Hz}, 1 \mathrm{H})$. MS: $m / z(\%) 261(100)[\mathrm{M}+1]$.

1,2:5,6-Di-O-isopropylidene-3-O-\{3'-(hydroxy)propyl $\}$-a-D-glucofuranose (2). A mixture of compound 1 (49.9 g, $192 \mathrm{mmol})$, 3-chloro-1-propanol (19.9 g, $211 \mathrm{mmol})$ and $\mathrm{NaOH}(23.0 \mathrm{~g}$, $576 \mathrm{mmol}$ ) was stirred at $110{ }^{\circ} \mathrm{C}$ for $8 \mathrm{~h}$. After cooling to $25-30{ }^{\circ} \mathrm{C}$, the reaction mixture was extracted with EtOAc $(2 \times 200 \mathrm{~mL})$. The combined organic layers were washed with water $(2 \times 100 \mathrm{~mL})$, brine $(1 \times 100 \mathrm{~mL})$, dried $\left(\mathrm{Na}_{2} \mathrm{SO}_{4}\right)$ and filtered. The filtrate was concentrated under reduced pressure to yield the crude product, which was purified by column chromatography over silica gel (100-200 mesh) using EtOAc-hexane (5:95) as eluent to give $\mathbf{2}$ as a colorless, thick oil, yield $52.1 \mathrm{~g}(85 \%) .{ }^{1} \mathrm{H} \mathrm{NMR}\left(\mathrm{CDCl}_{3}\right): \delta 1.39(\mathrm{~s}, 6 \mathrm{H}), 1.42(\mathrm{~s}, 6 \mathrm{H}), 1.80-1.92(\mathrm{~m}, 2 \mathrm{H}), 2.05(\mathrm{br} \mathrm{s}$, $1 \mathrm{H}), 3.48-3.61(\mathrm{~m}, 4 \mathrm{H}), 3.80-4.62(\mathrm{~m}, 6 \mathrm{H}), 5.84(\mathrm{~d}, J=4.0 \mathrm{~Hz}, 1 \mathrm{H}) . \mathrm{MS}: \mathrm{m} / z(\%) 319(100)$ [M+1]. Anal. Calcd for $\mathrm{C}_{15} \mathrm{H}_{26} \mathrm{O}_{7}$ (318.36): C, 56.59; H, 8.23\%. Found: C, 56.91; H, 7.96\%.

1,2:5,6-Di-O-isopropylidene-3-O-\{3'-(decyloxy)propyl\}- $\alpha$-D-glucofuranose (3). A mixture of compound 2 (50.0 g, $157.2 \mathrm{mmol})$, 1-bromodecane $(38.2 \mathrm{~g}, 172.9 \mathrm{mmol})$ and $\mathrm{NaOH}(18.9 \mathrm{~g}$, $471.6 \mathrm{mmol}$ ) was stirred at $110{ }^{\circ} \mathrm{C}$ for $8 \mathrm{~h}$. After cooling to $25-30{ }^{\circ} \mathrm{C}$, the reaction mixture was extracted with EtOAc $(2 \times 200 \mathrm{~mL})$. The combined organic layers were washed with water $(2 \times 100 \mathrm{~mL})$, brine $(1 \times 100 \mathrm{~mL})$, dried $\left(\mathrm{Na}_{2} \mathrm{SO}_{4}\right)$ and filtered. The filtrate was concentrated under reduced pressure to yield the crude product, which was purified by column chromatography over silica gel (100-200 mesh) using EtOAc-hexane (5:95) as eluent to give $\mathbf{3}$ as a colorless, thick oil, yield $62.1 \mathrm{~g}(86 \%) .{ }^{1} \mathrm{H}$ NMR $\left(\mathrm{CDCl}_{3}\right): \delta 0.95(\mathrm{t}, J=6.5 \mathrm{~Hz}, 3 \mathrm{H}), 1.24-1.60(\mathrm{~m}, 30 \mathrm{H}), 3.28-3.57$ $(\mathrm{m}, 6 \mathrm{H}), 3.80-4.65(\mathrm{~m}, 6 \mathrm{H}), 5.90(\mathrm{~d}, J=4.0 \mathrm{~Hz}, 1 \mathrm{H}) . \mathrm{MS}: \mathrm{m} / z(\%) 459(100)[\mathrm{M}+1]$. Anal. Calcd for $\mathrm{C}_{25} \mathrm{H}_{46} \mathrm{O}_{7}$ (458.63): C, 65.47; H, 10.11\%. Found: C, 65.76; H, 10.32\%.

3-O-\{3'-(Decyloxy)propyl\}-1,2-O-isopropylidene- $\alpha$-D-glucofuranose (4). To a stirred solution of compound $3(62.0 \mathrm{~g}, 135.4 \mathrm{mmol})$ in THF $(310 \mathrm{~mL})$ was added $30 \% \mathrm{HClO}_{4}(62 \mathrm{~mL})$ at $0{ }^{\circ} \mathrm{C}$. After completion of the addition, stirring was continued at the same temperature for $6 \mathrm{~h}$. The 
reaction mixture was neutralized with a saturated solution of $\mathrm{Na}_{2} \mathrm{CO}_{3}(\mathrm{pH} 9.0)$ and the compound was extracted with EtOAc $(2 \times 150 \mathrm{~mL})$. The combined EtOAc extracts were washed with water $(2 \times 100 \mathrm{~mL})$, brine $(1 \times 100 \mathrm{~mL})$, dried $\left(\mathrm{Na}_{2} \mathrm{SO}_{4}\right)$ and filtered. The filtrate was concentrated under reduced pressure to give a crude product, which was purified by column chromatography over silica gel (100-200 mesh) using EtOAc-hexane (1:1) as eluent to give 4, yield $50.15 \mathrm{~g} \mathrm{(89 \% ).}{ }^{1} \mathrm{H}$ NMR $\left(\mathrm{CDCl}_{3}\right): \delta 0.98(\mathrm{t}, J=6.5 \mathrm{~Hz}, 3 \mathrm{H}), 1.20-1.71(\mathrm{~m}, 24 \mathrm{H}), 3.30-3.60(\mathrm{~m}, 6 \mathrm{H}), 3.65-4.65(\mathrm{~m}$, $6 \mathrm{H}), 4.70(\mathrm{br} \mathrm{s}, 2 \mathrm{H}), 5.90(\mathrm{~d}, J=4.0 \mathrm{~Hz}, 1 \mathrm{H})$. MS: $m / z(\%) 419(100)[\mathrm{M}+1]$. Anal. Calcd for $\mathrm{C}_{22} \mathrm{H}_{42} \mathrm{O}_{7}$ (418.56): C, 63.13; H, 10.11\%. Found: C, 62.89; H, 9.99\%.

3-O-\{3'-(Decyloxy)propyl\}-1,2-O-isopropylidene-5-carboxaldehyde- $\alpha$-D-xylofuranose

To a stirred solution of diol $4(50.0 \mathrm{~g}, 119.6 \mathrm{mmol})$ in water $(100 \mathrm{~mL})$, was added a solution of $\mathrm{NaIO}_{4}(50.9 \mathrm{~g}, 239.2 \mathrm{mmol})$ in water $(100 \mathrm{~mL})$ at $0{ }^{\circ} \mathrm{C}$. After completion of the addition, stirring was continued at the same temperature for $6 \mathrm{~h}$. Ethanol $(500 \mathrm{~mL})$ was added to the reaction mixture and the precipitated salts were filtered off and washed with ethanol $(1 \times 50 \mathrm{~mL})$. The filtrate was concentrated under reduced pressure and the residue was dissolved in EtOAc $(100 \mathrm{~mL})$, dried $\left(\mathrm{Na}_{2} \mathrm{SO}_{4}\right)$ and filtered. The filtrate was concentrated under reduced pressure to give 5 as a colorless, thick oil, yield $40.2 \mathrm{~g}(87 \%) .{ }^{1} \mathrm{H} \mathrm{NMR}\left(\mathrm{CDCl}_{3}\right): \delta 0.90(\mathrm{t}, J=6.5 \mathrm{~Hz}, 3 \mathrm{H})$, $1.20-1.69(\mathrm{~m}, 24 \mathrm{H}), 3.33-3.65(\mathrm{~m}, 6 \mathrm{H}), 4.05-4.66(\mathrm{~m}, 3 \mathrm{H}), 6.05(\mathrm{~d}, J=4.0 \mathrm{~Hz}, 1 \mathrm{H}), 9.61$ (br s, 1H). MS: $m / z(\%) 387(100)[\mathrm{M}+1]$. Anal. Calcd for $\mathrm{C}_{21} \mathrm{H}_{38} \mathrm{O}_{6}(386.52)$ : C, 65.25; H, 9.91\%. Found: C, 65.31; H, 10.05\%.

3-O-\{3'-(Decyloxy)propyl\}-1,2-O-isopropylidene- $\alpha$-D-xylofuranose (6). To a stirred solution of aldehyde 5 (38.0 g, $98.4 \mathrm{mmol})$ in absolute $\mathrm{MeOH}(150 \mathrm{~mL})$ was added $\mathrm{NaBH}_{4}(5.5 \mathrm{~g}$, $147.6 \mathrm{mmol}$ ) in portions at $0{ }^{\circ} \mathrm{C}$. After completion of the addition, the reaction mixture was stirred at the same temperature for $6 \mathrm{~h}$. The excess $\mathrm{NaBH}_{4}$ was then decomposed by addition of acetone $(20 \mathrm{~mL})$ and the resulting solution was concentrated under reduced pressure. The residue was dissolved in EtOAc $(200 \mathrm{~mL})$ and washed with brine $(1 \times 50 \mathrm{~mL})$. The organic layer was dried $\left(\mathrm{Na}_{2} \mathrm{SO}_{4}\right)$, filtered and the filtrate was concentrated under reduced pressure to give 6 as a colorless thick oil, yield $34.1 \mathrm{~g}(89 \%)$. ${ }^{1} \mathrm{H} \mathrm{NMR}\left(\mathrm{CDCl}_{3}\right): \delta 0.92(\mathrm{t}, J=6.5 \mathrm{~Hz}, 3 \mathrm{H}), 1.20-1.64$ (m, 24H), 3.25-3.61 (m, 6H), 4.25-4.55 (m, 5H), 4.69 (br s, 1H), 5.90 (d, $J=4.0 \mathrm{~Hz}, 1 \mathrm{H})$. MS: $m / z(\%) 389$ (100) [M+1]. Anal. Calcd for $\mathrm{C}_{21} \mathrm{H}_{40} \mathrm{O}_{6}$ (388.54): C, 64.92; H, 10.38\%. Found: C, $65.12 ; \mathrm{H}, 10.59 \%$.

\section{3-O-\{3'-(Decyloxy)propyl\}-1,2-O-isopropylidene-5-O-\{2'-(pyrrolidin-1-yl)ethyl\}- $\alpha$-D-}

xylofuranose (7a). General procedure for the preparation of 7. A mixture of compound 6 (0.78 g, $2.0 \mathrm{mmol}), 1$-(2-chloroethyl)pyrrolidine hydrochloride $(0.37 \mathrm{~g}, 2.2 \mathrm{mmol})$ and $\mathrm{NaOH}$ $(0.32 \mathrm{~g}, 8.0 \mathrm{mmol})$ was stirred at $110{ }^{\circ} \mathrm{C}$ for $6 \mathrm{~h}$. The reaction mixture was cooled to $25-30{ }^{\circ} \mathrm{C}$ and then extracted with EtOAc $(2 \times 25 \mathrm{~mL})$. The combined EtOAc layers were washed with water $(1 \times 25 \mathrm{~mL})$, brine $(1 \times 25 \mathrm{~mL})$, dried $\left(\mathrm{Na}_{2} \mathrm{SO}_{4}\right)$ and filtered. The filtrate was concentrated under reduced pressure to give $7 \mathbf{a}$ as a colorless, thick oil, which was purified by column chromatography over silica gel (100-200 mesh) using EtOAc-hexane (7:3) as eluent, yield $0.64 \mathrm{~g}(66 \%)$. IR (neat): 3100, 2900, 2800, 1440, 1390, $1150 \mathrm{~cm}^{-1} .{ }^{1} \mathrm{H}$ NMR $\left(\mathrm{CDCl}_{3}\right): \delta 0.92(\mathrm{t}$, $J=6.5 \mathrm{~Hz}, 3 \mathrm{H}), 1.22-1.60(\mathrm{~m}, 24 \mathrm{H}), 1.72-1.79(\mathrm{~m}, 4 \mathrm{H}), 2.57-2.82(\mathrm{~m}, 6 \mathrm{H}), 3.34-3.65(\mathrm{~m}, 10 \mathrm{H})$, 
4.19-4.65 (m, 3H), $6.00(\mathrm{~d}, J=4.0 \mathrm{~Hz}, 1 \mathrm{H}) .{ }^{13} \mathrm{C} \mathrm{NMR}\left(\mathrm{CDCl}_{3}\right): \delta 99.1,97.9,79.7,72.2,70.2$, $69.4,66.5,64.9,54.3,51.8,32.5,32.0,31.0,30.3,30.0,28.6,26.6,23.5,23.1,14.0 . \mathrm{MS}: \mathrm{m} / z(\%)$ 486 (100) [M+1]. Anal. Calcd for $\mathrm{C}_{27} \mathrm{H}_{51} \mathrm{NO}_{6}$ (485.70): C, 66.77; H, 10.58; N, 2.88\%. Found: C, $66.98 ; \mathrm{H}, 10.69 ; \mathrm{N}, 3.07 \%$.

3-O-\{3'-(Decyloxy)propyl\}-1,2-O-isopropylidene-5-O-\{2'-(1-hexamethyleneimino)ethyl $\}-\alpha-$ D-xylofuranose (7b). Colorless, thick oil (119 mg, 58\%). IR (neat): 3120, 2850, 2700, 1410, 1370, $1130 \mathrm{~cm}^{-1} .{ }^{1} \mathrm{H}$ NMR $\left(\mathrm{CDCl}_{3}\right): \delta 0.95(\mathrm{t}, J=6.5 \mathrm{~Hz}, 3 \mathrm{H}), 1.18-1.52(\mathrm{~m}, 22 \mathrm{H}), 1.66-1.77$ (m, 10H), 2.60-2.66 (m, 2H), 2.73-2.80 (m, 4H), 3.33-3.70 (m, 10H), 4.20-4.62 (m, 3H), 5.89 $(\mathrm{d}, J=4.0 \mathrm{~Hz}, 1 \mathrm{H}) . \mathrm{MS}: m / z(\%) 514(100)[\mathrm{M}+1]$. Anal. Calcd for $\mathrm{C}_{29} \mathrm{H}_{55} \mathrm{NO}_{6}$ (513.75): C, $67.80 ; \mathrm{H}, 10.79 ; \mathrm{N}, 2.73 \%$. Found: C, 68.02; H, 10.93; N, 2.88\%.

3-O-\{3'-(Decyloxy)propyl\}-1,2-O-isopropylidene-5-O-\{2'-(piperidin-1-yl)ethyl\}- $\alpha$-D xylofuranose (7c). Colorless, thick oil (139 mg, 70\%). IR (neat): 3150, 2900, 2750, 1450, 1350, $1100 \mathrm{~cm}^{-1} .{ }^{1} \mathrm{H}$ NMR $\left(\mathrm{CDCl}_{3}\right): \delta 0.90(\mathrm{t}, J=6.5 \mathrm{~Hz}, 3 \mathrm{H}), 1.21-1.58(\mathrm{~m}, 28 \mathrm{H}), 1.66-1.70(\mathrm{~m}, 2 \mathrm{H}), 2.23-2.31(\mathrm{~m}$, $4 \mathrm{H}), 2.59-2.62(\mathrm{~m}, 2 \mathrm{H}), 3.35-3.68(\mathrm{~m}, 10 \mathrm{H}), 4.21-4.60(\mathrm{~m}, 3 \mathrm{H}), 5.90(\mathrm{~d}, J=4.0 \mathrm{~Hz}, 1 \mathrm{H})$. MS: $\mathrm{m} / z(\%) 500$ (100) [M+1]. Anal. Calcd for $\mathrm{C}_{28} \mathrm{H}_{53} \mathrm{NO}_{6}$ (499.72): C, 67.30; H, 10.69; N, 2.80\%. Found: C, 67.11; H, 10.37; N, 2.62\%.

3-O-\{3'-(Decyloxy)propyl\}-1,2-O-isopropylidene-5-O-\{3'-(piperazin-1-yl)propyl\}- $\alpha$-D-

xylofuranose (7d). Colorless, thick oil (121 mg, 59\%). IR (neat): 3200, 2950, 2800, 1440, 1390, $1150 \mathrm{~cm}^{-1} .{ }^{1} \mathrm{H} \mathrm{NMR}\left(\mathrm{CDCl}_{3}\right): \delta 0.92(\mathrm{t}, J=6.5 \mathrm{~Hz}, 3 \mathrm{H}), 1.02-1.65(\mathrm{~m}, 26 \mathrm{H}), 2.33-2.50(\mathrm{~m}$, $10 \mathrm{H}), 3.37-3.62(\mathrm{~m}, 11 \mathrm{H}), 4.29-4.56(\mathrm{~m}, 3 \mathrm{H}), 5.89(\mathrm{~d}, J=4.0 \mathrm{~Hz}, 1 \mathrm{H}) . \mathrm{MS}: m / z(\%) 515(100)$ $[\mathrm{M}+1]$. Anal. Calcd for $\mathrm{C}_{28} \mathrm{H}_{54} \mathrm{~N}_{2} \mathrm{O}_{6}(514.74)$ : C, 65.33; H, 10.57; N, 5.44\%. Found: $\mathrm{C}, 65.13 ; \mathrm{H}$, $10.25 ; \mathrm{N}, 5.30 \%$.

3-O-\{3'-(Decyloxy)propyl\}-1,2-O-isopropylidene-5-O-\{3'-(piperidin-1-yl)propyl\}-a-D xylofuranose (7e). Colorless, thick oil (123 mg, 60\%). IR (neat): 3150, 2910, 2805, 1430, 1390, $1160 \mathrm{~cm}^{-1} .{ }^{1} \mathrm{H}$ NMR $\left(\mathrm{CDCl}_{3}\right): \delta 0.92(\mathrm{t}, J=6.5 \mathrm{~Hz}, 3 \mathrm{H}), 1.19-1.68(\mathrm{~m}, 32 \mathrm{H}), 2.16-2.23(\mathrm{~m}, 4 \mathrm{H})$, 2.51-2.56 (m, 2H), 3.38-3.60 (m, 10H), 4.19-4.60 (m, 3H), $5.85(\mathrm{~d}, J=4.0 \mathrm{~Hz}, 1 \mathrm{H})$. MS: $m / z(\%) 514$ (100) [M+1]. Anal. Calcd for $\mathrm{C}_{29} \mathrm{H}_{55} \mathrm{NO}_{6}$ (513.75): C, 67.80; H, 10.79; N, 2.73\%. Found: C, 67.99; H, 10.86; N, 2.78\%.

3-O-\{3'-(Decyloxy)propyl\}-1,2-O-isopropylidene-5-O-\{3'-(1-phenyl)propyl\}- $\alpha$-D-xylofuranose (7f). Colorless, thick oil (109 mg, 54\%). IR (neat): 3200, 2960, 2830, 1410, 1360, $1140 \mathrm{~cm}^{-1} .{ }^{1} \mathrm{H}$ NMR $\left(\mathrm{CDCl}_{3}\right): \delta 0.93(\mathrm{t}, J=6.5 \mathrm{~Hz}, 3 \mathrm{H}), 1.20-1.55(\mathrm{~m}, 24 \mathrm{H}), 1.62-1.70(\mathrm{~m}, 2 \mathrm{H}), 2.84-2.92(\mathrm{~m}$, 2H), 3.36-3.80 (m, 10H), 4.31-4.58 (m, 3H), $5.90(\mathrm{~d}, J=4.0 \mathrm{~Hz}, 1 \mathrm{H}), 7.00-7.20(\mathrm{~m}, 5 \mathrm{H})$. MS: $m / z(\%) 507$ (100) [M+1]. Anal. Calcd for $\mathrm{C}_{30} \mathrm{H}_{50} \mathrm{O}_{6}$ (506.71): C, 71.11; H, 9.95\%. Found: C, $70.78 ; \mathrm{H}, 9.69 \%$.

33-O-\{3'-(Decyloxy)propyl\}-1,2-O-isopropylidene-5-O-\{2'-(morpholin-1-yl)ethyl\}- $\alpha$ - D-xylofuranose (7g). Colorless, thick oil (125 mg, 63\%). IR (neat): 3100, 2905, 2810, 1450, 1380, $1150 \mathrm{~cm}^{-1} .{ }^{1} \mathrm{H}$ NMR $\left(\mathrm{CDCl}_{3}\right): \delta 0.94(\mathrm{t}, J=6.5 \mathrm{~Hz}, 3 \mathrm{H}), 1.20-1.65(\mathrm{~m}, 24 \mathrm{H}), 2.28-2.60(\mathrm{~m}, 6 \mathrm{H}), 3.24-3.64(\mathrm{~m}$, $14 \mathrm{H}), 4.19-4.65(\mathrm{~m}, 3 \mathrm{H}), 5.92(\mathrm{~d}, J=4.0 \mathrm{~Hz}, 1 \mathrm{H}) . \mathrm{MS}: \mathrm{m} / z(\%) 502$ (100) [M+1]. Anal. Calcd for $\mathrm{C}_{27} \mathrm{H}_{51} \mathrm{NO}_{7}$ (501.70): C, 64.64; H, 10.25; N, 2.79\%. Found: C, 64.39; H, 10.01; N, 2.54\%. 
3-O-\{3'-(Decyloxy)propyl\}-1,2-O-isopropylidene-5-O- $\left\{3^{\prime}-\left(N^{\prime}, N^{\prime}\right.\right.$-dimethylamino)propyl $\}-\alpha-$ D-xylofuranose (7h). Colorless, thick oil (110 mg, 58\%). IR (neat): 3200, 2950, 2830, 1440, 1390, $1120 \mathrm{~cm}^{-1} .{ }^{1} \mathrm{H}$ NMR $\left(\mathrm{CDCl}_{3}\right): \delta 0.96(\mathrm{t}, J=6.5 \mathrm{~Hz}, 3 \mathrm{H}), 1.19-1.59(\mathrm{~m}, 22 \mathrm{H}), 1.67-1.70$ (m, 4H), 2.00-2.39 (m, 8H), 3.40-3.60 (m, 10H), 4.19-4.59 (m, 3H), $5.88(\mathrm{~d}, J=4.0 \mathrm{~Hz}, 1 \mathrm{H})$. MS: $m / z(\%) 474(100)[\mathrm{M}+1]$. Anal. Calcd for $\mathrm{C}_{26} \mathrm{H}_{51} \mathrm{NO}_{6}$ (473.69): C, 65.93; H, 10.85; N, 2.96\%. Found: C, 66.22; H, 10.97; N, 3.23\%.

3-O-\{3'-(Decyloxy)propyl\}-1,2-O-isopropylidene-5-O-p-tosyl- $\alpha$-D-xylofuranose (8). To a solution of alcohol $6(17.0 \mathrm{~g}, 43.8 \mathrm{mmol})$ in pyridine $(50 \mathrm{~mL})$, was added a solution of $p$ toluenesulphonyl chloride $(8.42 \mathrm{~g}, 44.2 \mathrm{mmol})$ in pyridine $(25 \mathrm{~mL})$ dropwise at $0{ }^{\circ} \mathrm{C}$ with stirring. After complete addition, the reaction mixture was stirred for $6 \mathrm{~h}$ at the same temperature. After completion of the reaction (TLC evidence), the solvent was removed under reduced pressure and the residue was dissolved in EtOAc $(100 \mathrm{~mL})$, washed with water $(2 \times 20 \mathrm{~mL})$ and a saturated solution of $\mathrm{NaHCO}_{3}(2 \times 20 \mathrm{~mL})$, dried $\left(\mathrm{Na}_{2} \mathrm{SO}_{4}\right)$ and filtered. The filtrate was concentrated under reduced pressure to give 8 as a viscous oil, yield $20.1 \mathrm{~g}(85 \%)$. ${ }^{1} \mathrm{HNMR}\left(\mathrm{CDCl}_{3}\right): \delta 0.93(\mathrm{t}, J=6.5 \mathrm{~Hz}, 3 \mathrm{H}), 1.21-1.60(\mathrm{~m}, 22 \mathrm{H}), 1.66-1.70(\mathrm{~m}, 2 \mathrm{H}), 2.35(\mathrm{~s}$, $3 \mathrm{H}), 3.36-3.72(\mathrm{~m}, 8 \mathrm{H}), 4.21-4.61(\mathrm{~m}, 3 \mathrm{H}), 5.95(\mathrm{~d}, J=4.0 \mathrm{~Hz}, 1 \mathrm{H}), 7.24-7.60(\mathrm{~m}, 4 \mathrm{H}) . \mathrm{MS}: \mathrm{m} / z$ (\%) 543 (100) $[\mathrm{M}+1]$. Anal. Calcd for $\mathrm{C}_{28} \mathrm{H}_{46} \mathrm{O}_{8} \mathrm{~S}$ (542.73): C, 61.96; H, 8.54\%. Found: C, $61.88 ; \mathrm{H}, 8.61 \%$.

\section{3-O-\{3'-(Decyloxy)propyl\}-1,2-O-isopropylidene-5-deoxy-5-(pyrrolidin-1-yl)- $\alpha$-D-xylofuranose}

(9a). General procedure for the preparation of 9. A mixture of compound 8 (1.0 g, $1.85 \mathrm{mmol})$ and pyrrolidine $(1.0 \mathrm{~mL})$ was heated at $70{ }^{\circ} \mathrm{C}$ for $6 \mathrm{~h}$. The excess of pyrrolidine was removed under reduced pressure and the residue was dissolved in EtOAc $(25 \mathrm{~mL})$. The EtOAc layer was washed with a saturated solution of $\mathrm{NaHCO}_{3}(1 \times 20 \mathrm{~mL})$, brine $(2 \times 10 \mathrm{~mL})$, dried $\left(\mathrm{Na}_{2} \mathrm{SO}_{4}\right)$ and filtered. The filtrate was concentrated under reduced pressure to give a crude product, which was purified by column chromatography over silica gel (100-200 mesh) using EtOAc-hexane (7:3) as eluent to give 9a as a colorless, thick oil, yield $0.60 \mathrm{~g}$ (74\%). IR (neat): 3300, 3100, 2900, 2800, 1650, 1440, 1390, 1350, $1150 \mathrm{~cm}^{-1} .{ }^{1} \mathrm{H}$ NMR $\left(\mathrm{CDCl}_{3}\right): \delta 0.95(\mathrm{t}, J=$ $6.5 \mathrm{~Hz}, 3 \mathrm{H}), 1.26-1.61(\mathrm{~m}, 24 \mathrm{H}), 1.73-1.81(\mathrm{~m}, 4 \mathrm{H}), 2.49-2.61(\mathrm{~m}, 6 \mathrm{H}), 3.40-3.60(\mathrm{~m}, 6 \mathrm{H})$, 4.16-4.76 (m, 3H), $5.95(\mathrm{~d}, J=4.0 \mathrm{~Hz}, 1 \mathrm{H}) .{ }^{13} \mathrm{C} \mathrm{NMR}\left(\mathrm{CDCl}_{3}\right): \delta 98.8,97.9,79.4,73.6,70.2$, 69.2, 66.5, 64.9, 53.1, 52.1, 32.5, 32.0, 31.0, 30.3, 28.6, 26.6, 23.5, 23.1, 14.0. MS: $m / z(\%) 442$ (100) $[\mathrm{M}+1]$. Anal. Calcd for $\mathrm{C}_{25} \mathrm{H}_{47} \mathrm{NO}_{5}$ (441.64): C, 67.99; H, 10.73; N, 3.17\%. Found: C, 68.12; H, 10.94; N, 3.41\%.

3-O-\{3'-(Decyloxy)propyl\}-1,2-O-isopropylidene-5-deoxy-5-(piperidin-1-yl)- $\alpha$-D-xylofuranose (9b). Colorless, thick oil (136 mg, 81\%). IR (neat): 3360, 3150, 2920, 2840, 1595, 1420, 1380, 1290, $1110 \mathrm{~cm}^{-1} .{ }^{1} \mathrm{H}$ NMR $\left(\mathrm{CDCl}_{3}\right): \delta 0.95(\mathrm{t}, J=6.5 \mathrm{~Hz}, 3 \mathrm{H}), 1.24-1.70(\mathrm{~m}, 30 \mathrm{H}), 2.28-2.60$ (m, 6H), 3.40-3.64 (m, 6H), 4.11-4.67 (m, 3H), 5.95 (d, J=4.0Hz, 1H). MS: m/z (\%) $456(100)$ $[\mathrm{M}+1]$. Anal. Calcd for $\mathrm{C}_{26} \mathrm{H}_{49} \mathrm{NO}_{5}$ (455.67): C, 68.53; H, 10.84; N, 3.07\%. Found: C, 68.19; H, 10.63 ; N, 2.82\%.

3-O-\{3'-(Decyloxy)propyl\}-1,2-O-isopropylidene-5-deoxy-5-(morpholin-1-yl)- $\alpha$-D-xylofuranose

(9c). Colorless, thick oil (138 mg, 81\%). IR (neat): 3320, 3105, 2900, 2850, 1620, 1410, 1350, 
1290, $1140 \mathrm{~cm}^{-1} .{ }^{1} \mathrm{H}$ NMR $\left(\mathrm{CDCl}_{3}\right): \delta 0.96(\mathrm{t}, J=6.5 \mathrm{~Hz}, 3 \mathrm{H}), 1.24-1.62(\mathrm{~m}, 24 \mathrm{H}), 2.34-2.63$ $(\mathrm{m}, 6 \mathrm{H}), 3.40-3.75(\mathrm{~m}, 10 \mathrm{H}), 4.19-4.70(\mathrm{~m}, 3 \mathrm{H}), 5.90(\mathrm{~d}, J=4.0 \mathrm{~Hz}, 1 \mathrm{H}) . \mathrm{MS}: m / z(\%) 458$ (100) $[\mathrm{M}+1]$. Anal. Calcd for $\mathrm{C}_{25} \mathrm{H}_{47} \mathrm{NO}_{6}$ (457.64): C, 65.61; H, 10.35; N, 3.06\%. Found: C, $65.89 ; \mathrm{H}, 10.41 ; \mathrm{N}, 3.17 \%$.

3-O-\{3'-(Decyloxy)propyl\}-1,2-O-isopropylidene-5-deoxy-5-\{2'-(1-hexamethyleneimino)ethylamino\}- $\alpha$-D-xylofuranose (9d). Colorless, thick oil (155 mg, 82\%). IR (neat): 3350, 3110, 2900, 2805, 1610, 1420, 1390, 1310, $1130 \mathrm{~cm}^{-1} .{ }^{1} \mathrm{H} \mathrm{NMR}\left(\mathrm{CDCl}_{3}\right): \delta 0.96(\mathrm{t}, J=6.5 \mathrm{~Hz}, 3 \mathrm{H})$, $1.30-1.45(\mathrm{~m}, 22 \mathrm{H}), 1.61-1.89(\mathrm{~m}, 11 \mathrm{H}), 2.50-2.89(\mathrm{~m}, 10 \mathrm{H}), 3.41-3.60(\mathrm{~m}, 6 \mathrm{H}), 4.19-4.70(\mathrm{~m}$, $3 \mathrm{H}), 5.80(\mathrm{~d}, J=4.0 \mathrm{~Hz}, 1 \mathrm{H})$. MS: $m / z(\%) 513(100)[\mathrm{M}+1]$. Anal. Calcd for $\mathrm{C}_{29} \mathrm{H}_{56} \mathrm{~N}_{2} \mathrm{O}_{5}$ (512.77): C, 67.93; H, 11.01; N, 5.46\%. Found: C, 68.28; H, 10.84; N, 5.71\%.

\section{3-O-\{3'-(Decyloxy)propyl\}-1,2-O-isopropylidene-5-deoxy-5-\{3'-( $N^{\prime}, N^{\prime}$-dimethylamino)-}

propylamino\}-a-D-xylofuranose (9e). Colorless, thick oil (136 mg, 78\%). IR (neat): 3330, 3110, 2900, 2850, 1650, 1430, 1400, 1330, $1150 \mathrm{~cm}^{-1} .{ }^{1} \mathrm{H} \mathrm{NMR}\left(\mathrm{CDCl}_{3}\right): \delta 0.95(\mathrm{t}, J=6.5 \mathrm{~Hz}$, $3 \mathrm{H}), 1.32-1.64(\mathrm{~m}, 26 \mathrm{H}), 2.10(\mathrm{~s}, 6 \mathrm{H}), 2.20-2.88(\mathrm{~m}, 7 \mathrm{H}), 3.39-3.70(\mathrm{~m}, 6 \mathrm{H}), 4.21-4.68(\mathrm{~m}$, $3 \mathrm{H}), 5.95(\mathrm{~d}, J=4.0 \mathrm{~Hz}, 1 \mathrm{H})$. MS: $m / z(\%) 473(100)[\mathrm{M}+1]$. Anal. Calcd for $\mathrm{C}_{26} \mathrm{H}_{52} \mathrm{~N}_{2} \mathrm{O}_{5}$ (472.70): C, 66.06; H, 11.09; N, 5.93\%. Found: C, 66.33; H, 10.91; N, 5.75\%.

3-O-\{3'-(Decyloxy)propyl\}-1,2-O-isopropylidene-5-deoxy-5-(heptylamino)- $\alpha$-D-xylofuranose (9f). Colorless, thick oil (120 mg, 67\%). IR (neat): 3300, 3105, 2900, 2850, 1600, 1430, 1385, 1350, $1130 \mathrm{~cm}^{-1} .{ }^{1} \mathrm{H}$ NMR $\left(\mathrm{CDCl}_{3}\right): \delta 0.90-1.20(\mathrm{~m}, 6 \mathrm{H}), 1.39-1.78(\mathrm{~m}, 35 \mathrm{H}), 2.48-2.82(\mathrm{~m}$, $4 \mathrm{H}), 3.53-3.75(\mathrm{~m}, 6 \mathrm{H}), 3.85-4.60(\mathrm{~m}, 3 \mathrm{H}), 5.74(\mathrm{~d}, J=4.0 \mathrm{~Hz}, 1 \mathrm{H}) . \mathrm{MS}: m / z(\%) 486(100)$ [M+1]. Anal. Calcd for $\mathrm{C}_{28} \mathrm{H}_{55} \mathrm{NO}_{5}$ (485.74): C, 69.23; H, 11.41; N, 2.88\%. Found: C, 68.96; H, $11.09 ; \mathrm{N}, 2.57 \%$.

3-O-\{3'-(Decyloxy)propyl\}-1,2-O-isopropylidene-5-deoxy-5-\{3'-(hydroxy)propylamino\}- $\alpha$ D-xylofuranose (9g). Colorless, thick oil (106 mg, 64\%). IR (neat): 3360, 3120, 2910, 2800, 1620, 1440, 1390, 1310, $1100 \mathrm{~cm}^{-1} .{ }^{1} \mathrm{H}$ NMR $\left(\mathrm{CDCl}_{3}\right): \delta 0.96(\mathrm{t}, J=6.5 \mathrm{~Hz}, 3 \mathrm{H}), 1.33-1.77(\mathrm{~m}$, 26H), 1.97 (br s, 2H) 2.68-2.90 (m, 4H), 3.38-3.70 (m, 8H), 3.80-4.58 (m, 3H), $5.76(\mathrm{~d}, J=$ 4.0Hz, 1H). MS: $m / z(\%) 446(100)[\mathrm{M}+1]$. Anal. Calcd for $\mathrm{C}_{24} \mathrm{H}_{47} \mathrm{NO}_{6}$ (445.63): C, 64.68; $\mathrm{H}$, 10.63 ; N, 3.14\%. Found: C, 64.89; H, 10.47; N, 3.01\%.

3-O-\{3'-(Decyloxy)propyl\}-1,2-O-isopropylidene-5-deoxy-5-\{2'-(1-morpholinyl) ethylamino\}- $\alpha$ D-xylofuranose (9h). Colorless, thick oil (138 mg, 74\%). IR (neat): 3330, 3150, 2900, 2830, 1600, 1410, 1385, 1350, $1150 \mathrm{~cm}^{-1} .{ }^{1} \mathrm{H} \mathrm{NMR}\left(\mathrm{CDCl}_{3}\right): \delta 0.94(\mathrm{t}, J=6.5 \mathrm{~Hz}, 3 \mathrm{H}), 1.24-1.63(\mathrm{~m}$, $24 \mathrm{H}), 1.88$ (br s, 1H), 2.52-2.86 (m, 10H), 3.41-3.71 (m, 10H), 4.19-4.61 (m, 3H), $5.95(\mathrm{~d}, J=$ 4.0Hz, 1H). MS: $m / z(\%) 501(100)[\mathrm{M}+1]$. Anal. Calcd for $\mathrm{C}_{27} \mathrm{H}_{52} \mathrm{~N}_{2} \mathrm{O}_{6}$ (500.71): C, 64.77; H, 10.47 ; N, 5.59\%. Found: C, 64.48; H, 10.34; N, 5.30\%.

3-O-\{3'-(Decyloxy)propyl\}-1,2-O-isopropylidene-5-deoxy-5-\{2'-(1-methylpyrrol-2-yl)ethylamino\}$\boldsymbol{\alpha}$-D- xylofuranose (9i). Colorless, thick oil (115 mg, 63\%). IR (neat): 3310, 3105, 2900, 2820, 1590, 1400, 1370, 1280, $1100 \mathrm{~cm}^{-1} .{ }^{1} \mathrm{H}$ NMR $\left(\mathrm{CDCl}_{3}\right): \delta 0.92(\mathrm{t}, J=6.5 \mathrm{~Hz}, 3 \mathrm{H}), 1.23-1.63(\mathrm{~m}$, 24H), 2.67-2.85 (m, 6H), 2.89 (br s, 1H) 3.40-3.71 (m, 9H), 4.19-4.68 (m, 3H), 5.95 (d, $J=$ $4.0 \mathrm{~Hz}, 1 \mathrm{H}), 6.10-6.52(\mathrm{~m}, 3 \mathrm{H})$. MS: $m / z(\%) 495(100)[\mathrm{M}+1]$. Anal. Calcd for $\mathrm{C}_{28} \mathrm{H}_{50} \mathrm{~N}_{2} \mathrm{O}_{5}$ (494.71): C, 67.98; H, 10.19; N, 5.66\%. Found: C, 67.64; H, 9.86; N, 5.23\%. 
3-O-\{3'-(Decyloxy)propyl\}-1,2-O-isopropylidene-5-deoxy-5-\{2'-(1-pyrrolidinyl)-ethylamino\}$\boldsymbol{\alpha}$ - D-xylofuranose (9j). Colorless, thick oil (120 mg, 67\%). IR (neat): 3330, 3120, 2910, 2800, 1630, 1420, 1390, 1300, $1150 \mathrm{~cm}^{-1} .{ }^{1} \mathrm{H}$ NMR $\left(\mathrm{CDCl}_{3}\right): \delta 0.94(\mathrm{t}, J=6.5 \mathrm{~Hz}, 3 \mathrm{H}), 1.30-1.54(\mathrm{~m}$, 24H), 1.61-1.88 (m, 7H), 2.50-2.82 (m, 8H), 3.41-3.73 (m, 6H), 4.19-4.68 (m, 3H), 5.95 (d, $J=$ 4.0Hz, 1H). MS: $m / z(\%) 485(100)[\mathrm{M}+1]$. Anal. Calcd for $\mathrm{C}_{27} \mathrm{H}_{52} \mathrm{~N}_{2} \mathrm{O}_{5}$ (484.71): C, 66.90; $\mathrm{H}$, $10.81 ; \mathrm{N}, 5.78 \%$. Found: C, 67.11; H, 11.08; N, 5.99\%.

3-O-\{3'-(Decyloxy)propyl\}-1,2-O-isopropylidene-5-deoxy-5-\{3'-(1-pyrrolidinyl)-propylamino\}$\boldsymbol{\alpha}$-D-xylofuranose (9k). Colorless, thick oil (118 mg, 64\%). IR (neat): 3350, 3105, 2920, 2850, 1610, 1440, 1370, 1280, $1100 \mathrm{~cm}^{-1} .{ }^{1} \mathrm{H}$ NMR $\left(\mathrm{CDCl}_{3}\right): \delta 0.94(\mathrm{t}, J=6.5 \mathrm{~Hz}, 3 \mathrm{H}), 1.30-1.54(\mathrm{~m}$, 24H), 1.61-1.88 (m, 7H), 2.50-2.82 (m, 10H), 3.41-3.72 (m, 6H), 4.21-4.70 (m, 3H), 5.95 (d, $J$ $=4.0 \mathrm{~Hz}, 1 \mathrm{H})$. MS: $m / z(\%) 499(100)[\mathrm{M}+1]$. Anal. Calcd for $\mathrm{C}_{28} \mathrm{H}_{54} \mathrm{~N}_{2} \mathrm{O}_{5}$ (498.74): C, 67.43; H, $10.91 ; \mathrm{N}, 5.62 \%$. Found: C, 67.32; H, 11.01; N, 5.77\%.

3-O-\{3'-(Decyloxy)propyl\}-1,2-O-isopropylidene-5-deoxy-5-\{2'-(1-piperidinyl)ethylamino\}$\boldsymbol{\alpha}$-D-xylofuranose (91). Colorless, thick oil (137 mg, 74\%). IR (neat): 3340, 2990, 2900, 2780, 1640, 1400, 1390, 1350, $1150 \mathrm{~cm}^{-1} .{ }^{1} \mathrm{H}$ NMR $\left(\mathrm{CDCl}_{3}\right): \delta 0.94(\mathrm{t}, J=6.5 \mathrm{~Hz}, 3 \mathrm{H}), 1.31-1.48(\mathrm{~m}$, 22H), 1.58-1.90 (m, 9H), 2.51-2.86 (m, 10H), 3.39-3.70 (m, 6H), 4.18-4.72 (m, 3H), $5.90(\mathrm{~d}, J$ $=4.0 \mathrm{~Hz}, 1 \mathrm{H})$. MS: $m / z(\%) 499(100)[\mathrm{M}+1]$. Anal. Calcd for $\mathrm{C}_{28} \mathrm{H}_{54} \mathrm{~N}_{2} \mathrm{O}_{5}$ (498.74): C, 67.43; H, 10.91 ; N, 5.62\%. Found: C, 67.61; H, 10.66; N, 5.40\%.

3-O-\{3'-(Decyloxy)propyl\}-1,2-O-isopropylidene-5-deoxy-5-\{3'-(1-imidazolyl)-propyl

amino\}- $\boldsymbol{\alpha}$-D-xylofuranose (9m). Colorless, thick oil (138 mg, 75\%). IR (neat): 3310, 3105, 2910, 28005, 1610, 1450, 1390, 1320, $1110 \mathrm{~cm}^{-1} .{ }^{1} \mathrm{H}$ NMR $\left(\mathrm{CDCl}_{3}\right): \delta 0.96(\mathrm{t}, J=6.5 \mathrm{~Hz}, 3 \mathrm{H})$, 1.35-1.76 (m, 25H), 2.05-2.45 (m, 2H), 2.80-3.85 (m, 6H), 3.90-4.15 (m, 6H), 4.20-4.59 (m, $3 \mathrm{H}), 5.65(\mathrm{~d}, J=4.0 \mathrm{~Hz}, 1 \mathrm{H}), 6.92-7.45(\mathrm{~m}, 3 \mathrm{H})$. MS: $m / z(\%) 496(100)[\mathrm{M}+1]$. Anal. Calcd for $\mathrm{C}_{27} \mathrm{H}_{49} \mathrm{~N}_{3} \mathrm{O}_{5}$ (495.70): C, 65.42; H, 9.96; N, 8.48\%. Found: C, 65.76; H, 10.13; N, 8.54\%.

\section{Acknowledgements}

We wish to express our thanks to the Analytical Chemistry Department of New Chemical Entity Research, Lupin Research Park, Pune for IR, ${ }^{1} \mathrm{H}$ NMR, mass spectroscopy, and elemental analyses of compounds synthesized.

\section{References}

1. Wolf, M. E. Berger's Medicinal Chemistry and Drug Discovery, John Wiley \& Sons: New York, 1995; Vol. 1, p 935.

2. Arora, S. K.; Schied, P. J. US Patent 1994, 5367 062; Chem. Abstr. 1995, 123, 228768.

3. Paul, G. Ger. Offen. 1975, 2455026; Chem. Abstr. 1975, 83, 147697e. 
4. Arora, S. K.; Gupta, M. K.; Lukos, P.; Kumar, R.; Sawhney, S. N. US Patent 1997, 5637570; Chem. Abstr. 1997, 126, 42715.

5. Takagi, Y.; Nakai, K. T.; Tsuchiya, T. T. J. Med. Chem. 1996, 39, 1582.

6. White, F. R. Cancer Chemother. Rep. 1963, 30, 49.

7. Evans, J. S.; Gerritsen, G. C.; Mann, K. M.; Owen, S. P. Cancer Chemother. Rep. 1965, 48, 1 .

8. Bhuyan, B. K.; Fraser, T. J.; Buskirk, H. H.; Neil, G. L. Cancer Chemother. Rep. 1972, 56, 709.

9. Fiebig, H. H.; Widmer, K. H.; Winterhalter, B. R.; Lohr, G. W. Cancer Chemother. Pharmacol. 1989, 23, 337.

10. Oachi, T.; Satoshi, J.; Fujie, H.; Chikashita, H. J. Heterocyclic Chem. 1984, 21, 1023.

11. Rosenthal, A.; Mikhailov, S. N. Carbohyds. Res. 1980, 79, 235.

12. Rosowsky, A.; Lazarus, H.; Yamashita, A. J. Med. Chem. 1976, 19, 1265.

13. Matsuda, A.; Takenuki, K.; Sasaki, T.; Ueda, T. J. Med. Chem. 1991, 34, 234.

14. Hottori, H.; Tanaka, M.; Fukushima, M.; Sasaki, T.; Matsuda, A. J. Med. Chem. 1996, 39, 5005.

15. Walton, E.; Jenkins, S. R.; Nutt, R. F.; Holly, F. W. J. Med. Chem. 1969, 12, 306.

16. Kishore, N.; Jain, S.; Sinha, N.; Upadhayaya, R. S.; Chandra, R.; Arora, S. K. ARKIVOC 2005, (iii), 156.

17. Furniss, B. S.; Hannaford, A. J.; Smith, P. W. G.; Tatchell, A. R. Vogel's Text Book of Practical Organic Chemistry; Addision Wesley Longman Limited: England, 1996; p 654.

18. (a) Grever, M. R.; Sherpartz, S. A.; Chabner, B. A. Semin. Oncol. 1992, 19, 622. (b) Monks, A. P.; Seudiero, D. A.; Skehan, P.; Shoemaker, R.; Paull, K. D.; Vistica, D.; Hose, C.; Langley, J.; Croniste, P.; Vaigro-Woiff, A.; Gray-Goodrich, M.; Campbell, H.; Mayo, J.; Boyd, M. R. J. Natl. Cancer Inst. 1991, 83, 757. (c) Weinstein, J. N.; Meyers, T. G.; O'Connor, P. M.; Friend, S. H.; Fornace, A. J., Jr.; Kohn, K. W.; Fojo, T.; Bates, S. E.; Rubinstein, L. V.; Anderson, N. L.; Boulamwini, J. K.; van Osdol, W. W.; Monks, A. P.; Seudiero, D. A.; Sausville, E. A.; Zaharevitz, D. W.; Bunow, B.; Viswanadhan, V. N.; Johnson, G. S.; Wittes, R. E.; Paull, K. D. Science 1997, 275, 343. 